\title{
Artículo
}

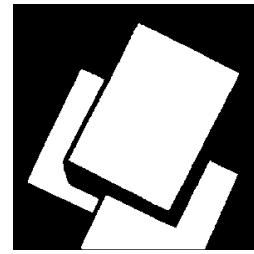

\section{La influencia de los rasgos psicológicos en las actitudes hacia el empleo}

Influence of psychological factors on work attitudes

ANTONIA MERCEDES GARCÍA CABRERA ${ }^{1}$ MARÍA GRACIA GARCÍA SOTO ${ }^{1}$

Fecha de Recepción: 16-01-2008 Fecha de Aceptación: 06-02-2009

\section{RESUMEN}

Esta investigación explica la influencia que el rasgo psicológico locus de control, conjuntamente con las variables que definen el capital humano de un país -género, edad y educación-, ejercen sobre las actitudes hacia el empleo en la República de Cabo Verde. De entre estos rasgos demográficos del individuo, el género es analizado más profundamente debido al interés actual por reducir las desigualdades en razón de género en el mercado laboral. Los 448 cuestionarios recabados muestran que, en este país, los atributos demográficos edad y nivel de estudios, así como el rasgo de personalidad locus de control, son importantes determinantes de las actitudes hacia el empleo de la población activa. Los resultados ofrecen las bases sobre las que redirigir las políticas de empleo en Cabo Verde para afrontar los puntos débiles que limitan el desarrollo del mercado de trabajo y amenazan la integración social.

\section{ABSTRACT}

This paper examines the influence of locus of control on work attitudes in terms of gender, age and education in the Republic of Cape Verde. Gender becomes the most relevant among those demographic variables according to the current emphasis on reducing genderrelated inequalities in the workplace. A number of 448 questionnaires were collected, showing that age, education level and locus of control are relevant factors in determining attitu-

\footnotetext{
${ }^{1}$ Facultad de ciencias Económicas y Empresariales. Universidad de Las Palmas de Gran Canaria.
} 
La influencia de los rasgos psicológicos en las actitudes hacia el empleo

des towards work. Results show the way to readdress employment policies in Cape Verde, especially weaknesses that restrict the development of the labor market and threaten social integration.

\section{PALABRAS CLAVE}

República de Cabo Verde, Mercado de trabajo, Actitudes hacia el trabajo, Emprendeduría, Locus de control.

\section{KEY WORDS}

Republic of Cape Verde, Labor Market, Work attitudes, Entrepreneurship, Locus of control. 


\section{INTRODUCCIÓN}

Son muchos los trabajos de investigación realizados sobre los factores que intervienen en el dinamismo del mercado de trabajo (i.e., trabajadores, empleadores, administración pública y sindicatos). En este estudio, centramos nuestro interés en el papel de los trabajadores como factor movilizador del comportamiento económico de un país. Ahora bien, las características del empleo y la forma en que las personas las perciben son aspectos íntimamente relacionados con la estructura social del territorio en que se inserta. De una forma más particular, Bauder (2006) señala que las actitudes hacia el empleo difieren entre sociedades y grupos sociales. Debido a ello, adquiere una gran relevancia la territorialización del estudio del mercado de trabajo, pues las políticas formuladas deben emanar de la idiosincrasia de cada lugar y, más específicamente, de los atributos característicos de la población activa.

En este contexto, la investigación pasada ha enfatizado el estudio de los rasgos demográficos y las competencias del individuo, surgiendo más recientemente la necesidad de incorporar los rasgos psicológicos como constructo adicional en el estudio de las actitudes hacia el trabajo. Ello es debido a que variables tales como el género, la edad o el nivel de estudios pueden ser insuficientes para entender las actitudes que el individuo adopta y condicionan su manera de afrontar la relación con el mercado de trabajo (Groves, 2005). En este sentido, merece destacar que si bien los rasgos psicológicos del individuo y, más particularmente, el locus de control, han sido extensamente contemplados en la literatura que investiga la predisposición a poner en marcha un negocio propio (e.g., Lee y Tsang, 2001; Mueller y Tho- mas, 2000; Premeaux y Bedeian, 2003; Thomas y Mueller, 2000), esta variable ha recibido poca atención cuando se investigan otras actitudes hacia el trabajo (e.g., predisposición a cambiar de oficio, emplearse a tiempo parcial), desconociéndose su efecto potencial y capacidad explicativa frente a los atributos característicos de la población activa tradicionalmente estudiados en la literatura (Becker y Hills, 1981; Becker y Krzystofiak, 1982; Frantz, 1980; Groves, 2005). Adicionalmente, las investigaciones empíricas se han llevado a cabo en economías occidentales desarrolladas, existiendo una laguna investigadora en el conocimiento de las variables explicativas del mercado laboral en una economía en transición.

Sobre la base de lo expuesto, planteamos como objetivo de investigación el análisis de la influencia que el rasgo psicológico locus de control ejerce sobre la actitud hacia el trabajo en una economía en transición, como es la República de Cabo Verde. De esta forma, la presente investigación permitirá escrutar la relevancia del locus de control como variable explicativa del mercado de trabajo en nuevos contextos geográficos y económicos hasta el momento no explorados.

Con el propósito de abordar el objetivo anteriormente planteado se lleva a cabo una revisión de la literatura sobre las actitudes hacia el mercado de trabajo y los factores que condicionan las mismas. Posteriormente, se describe Cabo Verde como contexto geográfico de estudio y se detalla la metodología utilizada. A este respecto, la información necesaria para llevar a cabo esta investigación fue recabada mediante un estudio empírico que permitió recopilar 448 cuestionarios válidamente cumplimentados en lengua portuguesa. Los resultados 
obtenidos nos permiten dar respuesta al objetivo de investigación formulado. Así, en primer lugar, se describen las características del empleo en el país objeto de estudio, las expectativas y actitudes hacia las condiciones del trabajo, el desarrollo de la emprendeduría, la movilidad territorial, y otros aspectos que condicionan la empleabilidad del individuo y el dinamismo del mercado de trabajo en la República de Cabo Verde. Y, en segundo lugar, más directamente relacionado con el objeto del presente trabajo, se analiza el valor del locus de control como variable explicativa de las actitudes hacia el trabajo.

\section{ACTITUDES HACIA EL TRABAJO Y DINAMISMO DEL MERCADO LABORAL}

La tasa de desempleo constituye el indicador más ampliamente utilizado para calibrar el bienestar del mercado laboral, así como una medida importante del estado de una economía en general (Byrne y Strobl, 2004). En economías en desarrollo, este indicador alcanza niveles muy elevados, siendo la lucha contra el desempleo prioridad tanto de los gobiernos de tales países (Harper, 1991) como de los organismos internacionales interesados en su desarrollo económico (e.g., Banco Mundial, Fondo Monetario Internacional, Banco para el Desarrollo Africano). En este sentido, la literatura publicada ha demostrado que el dinamismo del mercado de trabajo afecta positivamente a la productividad y al crecimiento económico de un país, efecto que es incluso más contundente en las economías en desarrollo (Poirson, 2001).

En este contexto, los factores que intervienen en el dinamismo del mercado de trabajo son diversos y, entre ellos, destacan, los empresarios, la administración pública, los sindicatos y el capital humano. En relación a este último, la investigación económica llevada a cabo ha sido extensa pues es a los individuos a quienes corresponde la adopción de medidas encaminadas a ampliar la propia empleabilidad. Concretamente, la empleabilidad es entendida en este trabajo como el conjunto de cualidades y actitudes hacia el trabajo que permiten al individuo permanecer como recurso atractivo en el mercado de trabajo (Sanders y de Grip, 2004), asumiendo una mayor movilidad ocupacional, así como un esquema de trabajo flexible (Kruss, 2004), por lo que generalmente tales actitudes conllevan un cierto sacrificio (Latack, 1986).

Las actitudes hacia el trabajo, más particularmente, hacen referencia al valor conferido por el individuo a ciertas actividades relacionadas con el mercado de trabajo, así como a los tipos de trabajo que se consideran prestigiosos, o que se estigmatizan, entre otros aspectos (Bauder, 2006). Ahora bien, estas actitudes de los individuos pueden variar, especialmente en aquellas circunstancias en las que surgen importantes oportunidades o limitaciones en el mercado de trabajo (Felfe, Schmook, Schyns y Six, 2008). El desempleo y el infraempleo, en este contexto, suelen requerir de los individuos la adopción de decisiones y el cambio de algunas de estas actitudes para superar tal situación. Entre tales actitudes hacia el mercado de trabajo, destacamos aquellas que son ampliamente reconocidas en la literatura por su contribución a la empleabilidad de la población activa: apertura a la movilidad geográfica (Gibson, 2005; Hanisch, 1999; Mcquaid, Greig y Adams, 2001) y ocupacional (Moscarini y Thomsson, 2007; Subrama- 
nian, 2008), compromiso con la formación (Lazarus y Folkman, 1984; Lee y Hsin, 2004; Sanders y de Grip, 2004), inclinación a combinar empleos múltiples a tiempo parcial (Bauder, 2006; Felfe et al., 2008; Hanisch, 1999), predisposición al empleo temporal (De Cuyper et al., 2008; Felfe et al., 2008; Yeh, Ko, Chang y Chen, 2007), aceptación del infraempleo (Bauder, 2006; Hanisch, 1999; Liem, 1992), o disposición a crear un negocio propio (Felfe et al., 2008; Galao Baptista y Vidigal da Silva, 2004).

Hanisch (1999) destaca, en primer lugar, la importancia de la reubicación geográfica (migración entre provincias y entre países) como factor que condiciona la empleabilidad del individuo. A nivel agregado, la movilidad geográfica afectaría positivamente al nivel de ajuste entre la demanda de empleo y la oferta de trabajo, reduciendo la tasa de desempleo en el territorio (Gabriel y Rosenthal, 1996). No obstante, en economías en transición, esta movilidad no debe constituir una migración masiva de las áreas rurales a las ciudades ya que esto conllevaría una menor probabilidad de que la población emigrante hallase empleo. En opinión de Gibson (2005), esta migración rural-urbana llevaría aparejada un círculo vicioso de estancamiento y pobreza para el territorio. Frente a ello, la reubicación geográfica debe ser entendida como la actitud o voluntad de los demandantes de empleo, o de aquéllos que desean mejorar su puesto actual, de trasladarse para hallar un empleo potencial en mercados con oferta creciente. En contraposición, la inflexibilidad de los individuos para trasladarse a otras regiones o lugares geográficos, debido, por ejemplo, a la propiedad o tenencia de una vivienda propia (Mcquaid et al., 2001), limita el número y tipo de oportu- nidades de empleo a que éstos pueden acceder (Ong y Blumenberg, 1998). Cuando esta última actitud prevalece en la población activa, se resiente tanto la empleabilidad de la misma, desde un ámbito personal, como la capacidad de la economía para atender la oferta de empleo en zonas demandantes del mismo.

La movilidad ocupacional, por otra parte, es destacada por los economistas debido a diversas razones entre las que destacan, desde el punto de vista del empleado, su clara relación con la búsqueda y acceso a oportunidades de empleo (Ferri, 2005); y, desde una perspectiva macroeconómica, su influencia positiva en la redistribución del capital humano entre sectores y ocupaciones para apoyar el crecimiento económico de un país (Moscarini y Thomsson, 2007), así como la posibilidad de recuperar perfiles ocupacionales específicos que en momentos de transición económica dejan de ser necesarios (Kambourov y Manovskii, 2002). A pesar de ello, el trabajo de Moscarini y Thomsson (2007) en Estados Unidos, pone de manifiesto que la movilidad ocupacional en las últimas dos décadas ha mostrado un comportamiento oscilante debido, entre otros factores, al hecho de que la incertidumbre percibida por la población activa podría hacer que los individuos adoptasen comportamientos más defensivos que conducen a la reducción de la movilidad ocupacional. En economías en transición, en las que la reactivación y el desarrollo económico del país representan un objetivo primordial, la actitud positiva del capital humano frente a la movilidad ocupacional parecen fundamentales.

La literatura sobre capital humano ha destacado ampliamente el papel de la formación como factor que mejora la emple- 
abilidad de la población activa y, más particularmente, las oportunidades de encontrar empleo tras estar en situación de paro (Lee y Hsin, 2004), así como de lograr la empleabilidad en otros oficios y facilitar la movilidad ocupacional (Lazarus y Folkman, 1984; Sanders y de Grip, 2004). No sorprende entonces que aquellos empleados que tienen la voluntad de permanecer como un recurso atractivo en el mercado laboral, de mejorar su empleabilidad o de incrementar el salario percibido, muestren una actitud favorable hacia la formación y el reciclaje, comprometiéndose en distintas actividades formativas (Sanders y de Grip, 2004). En términos agregados, un aprendizaje activo por parte de la población activa es especialmente importante en las situaciones de crisis, cambio e incertidumbre que acompañan a las economías en transición. Este aprendizaje contribuiría a potenciar en la población activa el desarrollo de competencias, habilidades y actitudes que mejoren tanto su posición como su contribución al mercado laboral, lo que es especialmente relevante debido a que los niveles formativos son significativamente bajos en estos países.

El empleo temporal, por otra parte, se caracteriza por el horizonte de tiempo limitado que se asocia a un puesto de trabajo desempeñado en una organización (Felfe et al., 2008). El crecimiento de esta forma de empleo en países industrializados (Ogura, 2005) ha sido exponencial en las últimas dos décadas (Slattery, Selvarajan y Anderson, 2008) y se prevé un incremento continuado, aunque a un ritmo más moderado en los próximos años (Guest, 2004). Ello ha llevado a algunos investigadores a referirse a esta modalidad de empleo como la evolución más espectacular e importante de la vida laboral en Occidente (De Cuyper et al., 2008). A este respecto, se ha destacado que el trabajo temporal aporta flexibilidad tanto al empleador (De Cuyper et al., 2008) como al empleado (Felfe et al., 2008). Ahora bien, junto a las ventajas que este tipo de empleo aporta al trabajador -e.g., autonomía, variabilidad, oportunidad de incrementar la experiencia- (Torka y Schyns, 2007), también existe un amplio conjunto de desventajas -e.g., salarios más bajos, menores oportunidades de promoción laboral, sentimiento de inseguridad, estrés laboral, etc.- (Yeh et al., 2007). Como resultado, el individuo habitualmente concibe al empleo temporal como una solución transitoria hasta encontrar un empleo indefinido (Torka y Schyns, 2007), no existiendo una actitud favorable en la población activa hacia esta modalidad de empleo en países occidentales. El debate planteado respecto a la actitud del empleado frente al empleo temporal en economías occidentales y desarrolladas, carece en cierta medida de relevancia en economías en transición, donde el problema del desarrollo relega, en cierta medida, la relevancia de su carácter temporal o fijo. De hecho, el empleo temporal es una práctica habitual y consolidada entre empleadores y empleados.

La decisión de recurrir a la emprendeduría o al autoempleo, por su parte, sí representa una importante actitud hacia el empleo en economías en transición (Galao Baptista y Vidigal da Silva, 2004). La iniciativa emprendedora, en estos contextos económicos, habitualmente incluye la emprendeduría informal, caracterizada por la existencia de un gran número de microempresas cuyos propietarios son familias que las dirigen y hacen uso de tecnologías sencillas (Todaro, 1997). La micro-empresa familiar se corresponde en este caso con un negocio de pequeño tamaño, nor- 
malmente sin empleados ajenos a la familia, que no se encuentra formalmente constituido. Específicamente, la investigación llevada a cabo en países en vías de desarrollo deja constancia de la existencia de un alto comportamiento emprendedor por parte de la población, tal y como se ha demostrado en los trabajos realizados en el marco del Global Entrepreneurship Monitor (Acs, Arenius, Hay y Minniti, 2005; Minniti, Bygrave y Autio, 2006; Reynolds, Bygrave, Autio, Cox y Hay, 2002). Esta investigación ha revelado la existencia de altos niveles de emprendeduría en los países de menores rentas, debido a que las opciones para emplearse son insatisfactorias o inexistentes (Acs et al., 2005). Este fenómeno es denominado por Verheul, Van Stel y Thurik (2006) como "emprendeduría por necesidad" pues surge del efecto "empuje" o "refugio" del desempleo (p. 156). La emprendeduría, en estos contextos económicos, no está basada en la vocación emprendedora; en su lugar, es una acción forzada por el desempleo o por la búsqueda de logro personal y reconocimiento social (Galao Baptista y Vidigal da Silva, 2004). De hecho, en estos países el número de empresas creadas por cada 100 adultos supera significativamente al existente en aquéllos de rentas más altas, donde las actividades emprendedoras sólo se llevan a cabo cuando permiten aprovechar oportunidades claramente identificables -i.e., emprendeduría oportunista- (Acs et al., 2005).

En situaciones en las que el mercado de trabajo está estancado, el empleo a tiempo parcial y el multiempleo que lleva asociado, constituyen formas alternativas de afrontar la relación con el mercado de trabajo, cada vez más relevantes y utilizadas (Felfe et al., 2008; Yeh et al., 2007). Cobra de esta forma especial relevancia que la población activa muestre su disposición a aceptar estas nuevas formas de empleo (Bauder, 2006; Hanisch, 1999). A este respecto, conviene destacar que, en ocasiones, el empleo a tiempo parcial da lugar al autoempleo, surgiendo así los denominados "semiemprendedores" que ostentan empleos múltiples, a saber, un trabajo por cuenta ajena a tiempo parcial y un pequeño negocio propio como medio para completar los menores ingresos que aporta el empleo parcial. Esta forma combinada de empleo puede llegar a ser común en las economías en transición en las que las iniciativas emprendedoras adoptan la forma de negocios informales ligados en muchos casos a actividades desarrolladas en entornos rurales (García Cabrera y García Soto, 2008).

Finalmente, la predisposición a ocupar puestos de trabajo en situación de infraempleo (e.g. no regulados, con salario inferior al que corresponde por formación, en categorías laborales inferiores a la que corresponde por nivel formativo, etc.) constituye otra posible decisión (Bauder, 2006; Hanisch, 1999) disponible para facilitar la empleabilidad. Ahora bien, estas actitudes y el empleo a que dan lugar, suelen llevar aparejadas insatisfacción, pérdida de autoestima y otros daños psicológicos en las personas que ocupan dichos puestos de trabajo (Liem, 1992). En opinión de Hanisch (1999), los individuos que acuden al infraempleo asumen unos costes psicológicos mayores al ocupar estos puestos de trabajo insatisfactorios (con condiciones teóricamente inaceptables) que los costes que les acarrearía estar desempleado. A pesar de ello, en entornos de bajo progreso económico, limitado desarrollo social y escasa legislación que regule el mercado de trabajo, es usual el desarrollo de una importante economía 
La influencia de los rasgos psicológicos en las actitudes hacia el empleo

sumergida que oferta puestos de trabajo no regulados y que representan una oportunidad para aquella población activa con actitudes favorables hacia el infraempleo.

Atendiendo a lo anteriormente expuesto, en la presente investigación se contemplan aquellas actitudes hacia el trabajo relevantes para una economía en transición, que incluyen la predisposición a cambiar de ocupación o de sector de actividad, la movilidad territorial, la disposición a participar en programas de actualización y reciclaje profesional, la predisposición a aceptar empleos en precario y mal regulados y la consideración del autoempleo (i.e., emprendeduría).

\section{FACTORES CONDICIONANTES DE LA ACTITUD HACIA EL TRABAJO}

Las actitudes hacia el trabajo están todas relacionadas con el perfil sociodemográfico del individuo y con su origen (Bauder, 2006). En este sentido, tanto la literatura interesada en mejorar el dinamismo del mercado de trabajo como aquélla otra orientada a identificar los factores que justifican un mayor éxito del individuo en el mismo (e.g., en términos de retribución o éxito profesional) se han centrado en analizar los efectos de las habilidades del individuo. Concretamente, la formación y la experiencia profesional son variables comúnmente analizadas (Groves, 2005). En opinión de Frantz (1980), una amplia literatura ha demostrado que estas formas de capital humano son instrumentales y facilitan la mejora de la empleabilidad y de los salarios por hora del trabajador. Adicionalmente, los factores género y edad, que constituyen parte relevante de la estructura social en la que el mercado de empleo se desenvuelve, también han sido objeto de tratamiento, pues constituyen atributos con capacidad para explicar el capital humano.

Sin embargo, buena parte de la varianza de los modelos explicativos del mercado de trabajo ha permanecido sin explicar lo que puede deberse a la no incorporación de los rasgos psicológicos de los individuos en dichos modelos (Groves, 2005). En este sentido, la corriente de investigación centrada en el estudio de las actitudes del empleado y su conexión con el mercado de trabajo, cuyo origen se remonta a los años ochenta, ha adquirido una importancia creciente. Dentro de esta línea, se consideran como activos valiosos del individuo a las variables de índole psicológica tales como, la motivación, la autoestima, la iniciativa del individuo o el locus de control, siendo este último un rasgo particularmente estudiado por su capacidad potencial para explicar el comportamiento y resultados en el mercado de trabajo (Becker y Hills, 1981; Becker y Krzystofiak, 1982; Frantz, 1980; Groves, 2005).

En este sentido, el locus de control es considerado un rasgo estable de la personalidad de los individuos (Levenson, 1974). Rotter (1966) originalmente lo definió como una creencia sobre la relación entre las acciones que uno realiza y el resultado real alcanzado. El locus de control interno se correspondería con la convicción personal de que los acontecimientos que se producen en la vida diaria son el resultado de la eficacia del comportamiento de cada individuo, de sus habilidades y de sus esfuerzos; el locus de control externo incluye la convicción de que tales resultados no dependen del propio individuo sino de la suerte, la oportunidad, el destino o el poder de otros. Este rasgo se 
conforma a medida que el individuo, desde la niñez, evoluciona y se desarrolla, recibe formación y se nutre de experiencias vitales, siendo este proceso y los factores que inciden en la conformación del locus (e.g., estatus social de los padres, acceso a la educación de los niños, tipo de educación que reciben, etc.) comúnmente analizados en la literatura (e.g., M. Omizo, Cubberly y S. Omizo, 1985; Stevens, Steffen, Dirroco y David, 2007). Sin embargo, a partir de la adolescencia, el locus de control se convierte en un rasgo estable de la personalidad del individuo (Levenson, 1974), difícilmente modificable. Al objeto de testar la posible influencia del mercado laboral sobre el locus de control en edad adulta, Frantz (1980) tras analizar el locus de control y la experiencia laboral, medida esta última a través del número de semanas trabajadas en un período de cuatro años, está en disposición de afirmar que ésta no tiene efectos significativos sobre el nivel de locus de control.

De forma más específica, el locus de control ha sido asociado con la decisión de inversión en capital humano, es decir, con la decisión individual de comprometer recursos y esfuerzos en un mayor enriquecimiento formativo (Capella y Weinstein, 2001; Coleman y Deleire, 2003; Mwamwenda, 1995), así como con las elecciones que en materia de formación realizan los individuos (Boone, Olffen y Roijakkers, 2004). Ello obedece a que cuando en el individuo predomina una creencia interna de control, existe la convicción personal de que los resultados de las acciones que él emprende dependen de la eficacia de su comportamiento, de sus habilidades y de sus esfuerzos. De esta forma, los individuos de locus interno tienen mayores expectativas sobre la rentabilidad futura a percibir como resultado de la inversión actual en formación (Coleman y Deleire, 2003), lo que justifica la asociación entre locus interno y mayores niveles de educación. Asimismo, el locus interno se encuentra asociado con mayor experiencia en el mercado de trabajo (Frantz, 1980) y con un mayor éxito en los procesos de transición ecológica que se produce cuando los individuos emigran en busca de empleo (Martínez García, García Ramírez y Maya Jariego, 2002). La asociación entre éxito en el mercado de trabajo y locus interno de control viene entonces motivada por el hecho de que los individuos de mayor control interno tienen una actitud más favorable hacia la adopción de decisiones personales tales como incrementar su formación, aceptar la movilidad de oficio o de residencia, entre otras. Estas actitudes, en opinión de Bauder (2006), tienen una influencia potencial en los resultados del individuo en el mercado de trabajo.

Lo hasta ahora expuesto nos lleva a adoptar una perspectiva integradora en el presente trabajo contemplando, en relación al capital humano, el estudio de aspectos tales como el género, la edad, el nivel educativo y el locus de control como rasgo de personalidad, dado que todas ellas se proyectan en las percepciones y actitudes hacia el empleo. Los argumentos anteriores también nos permite sustentar el presente trabajo en la premisa de que las variables demográficas y psicológicas características del capital humano determinan las actitudes hacia el trabajo por lo que se analiza dicha interacción para la República de Cabo Verde.

\section{LA REPÚBLICA DE CABO VERDE}

La República de Cabo Verde se encuentra ubicada a 450 Kilómetros de la 
costa de Senegal, por lo que es el país africano más alejado de su continente y más cercano al de América. Cabo Verde cuenta con 450.489 habitantes en 2002, ocupa una superficie de $4.033 \mathrm{~km}^{2}$, tiene escasos recursos minerales y sólo el 10 por ciento de su superficie es cultivable; disfruta de un clima tropical y semiárido por lo que padece de períodos frecuentes de sequías caracterizándose además su economía por estar escasamente industrializada. En términos económicos, destaca que el Producto Interior Bruto de este país osciló entre un 6 y un 4,5 por ciento entre 2002 y 2004. La renta per capita, sin embargo, no supera el 25 por ciento de la de países como España y Portugal, según consta en el Índice de Desarrollo Humano (2004) elaborado por la ONU, por lo que es calificado como un país de renta baja. Concretamente, Cabo verde se sitúa en el ranking de pobreza de los 56 países africanos en el número 38 , con un índice de pobreza del 19,7 por ciento.

Dado que las variables que definen el capital humano son el género, la estructura de edad y la educación, en el presente apartado se analizan brevemente las mismas para la República de Cabo Verde, haciendo hincapié, adicionalmente, en la desigualdad, por su relevancia para el normal desarrollo del mercado de trabajo. En primer lugar, y con respecto al género, destaca que en Cabo Verde las féminas residentes superan a los varones $(51,7 \%$ frente al 48,3\%) según US. Census Bureau (base de datos internacional). En lo que se refiere a la desigualdad en razón de género, Cabo Verde se adhiere en 1979, cuatro años después de conseguir la independencia de Portugal, a la Convención sobre la eliminación de todas las formas de discriminación con relación a las mujeres. Recibe, de esta forma, en su ordenamiento jurídico uno de los instrumentos que permitirán la aplicación de políticas que aseguran la igualdad entre los hombres y mujeres. La Constitución de la República explicita el principio de la igualdad y de la no discriminación por razón de sexo, lo que significa que hombres y mujeres disfrutan de iguales derechos, responsabilidades y obligaciones. De la misma forma, el Libro IV sobre la Familia y los Menores de la Ley Civil caboverdiana detalla tales derechos, responsabilidades y obligaciones. Adicionalmente, Cabo Verde ha participado en debates internacionales sobre la vulnerabilidad de la mujer y reconoce la necesidad de protección de este colectivo, lo que se refleja en los avances alcanzados en las disposiciones legales relacionadas con esta materia.

En el ámbito de la realidad cotidiana, sin embargo, la citada igualdad formal no se encuentra de facto respetada tanto en los ámbitos social como familiar, económico y político. Así, por ejemplo, en muchos casos existe discriminación laboral, de forma que por un mismo trabajo existe diferencia de sueldo, percibiendo las féminas salarios más bajos. Más concretamente, y dado que la legislación del país no permite la discriminación laboral, en la práctica se contrata a la mujer con categorías profesionales más bajas a la que le corresponde por su formación y por su experiencia laboral. Además, y en relación a la actividad, aunque el marco legal no reconoce oficios exclusivos para el hombre y para la mujer, socialmente algunas tareas son reservadas a las féminas. Finalmente, es necesario tener presente en esta investigación que, según el INE de Cabo Verde, el 40,1 por ciento de las familias caboverdianas tienen como cabeza de familia a mujeres. De hecho, la 
sociedad caboverdiana confiere a la mujer una responsabilidad casi sin límites con relación al sustento y manutención de la familia, siendo prácticamente la única responsable de la educación de los hijos. Ello obedece a que la poligamia masculina constituye una práctica habitual e institucionalizada, aunque ilegal y mal aceptada por parte de las mujeres. Estos antecedentes pueden justificar que la influencia del género en el mercado laboral no sea coincidente con la hallada en otros países.

Por otra parte, Cabo Verde es un país joven, tal y como refleja su pirámide poblacional. Los caboverdianos de edad inferior a 15 años se elevan al 41,9 por ciento del total, mientras que los comprendidos entre 15 y 34 años representan el 31,6 por ciento, frente a la población situada entre 35 y 64 años que no alcanza el 20 por ciento, y con más de 64 años que se reduce hasta el 6,6 por ciento (U.S. Census Bureau). Esta pirámide se corresponde con un modelo demográfico tradicional en el que destacan las elevadas tasas de natalidad y mortalidad fruto del limitado estado de desarrollo sociocultural de los países en transición. A ello se une una cultura económica sustentada en el apoyo familiar, en la que los diferentes miembros, independientemente de su edad, deben contribuir con su trabajo y apoyar la actividad profesional del cabeza de familia. Esto es común especialmente en las zonas rurales. Como resultado, las cifras estadísticas de población activa no siempre reflejan la realidad del país debido a que existe un porcentaje de caboverdianos implicados en actividades laborales que no se encuentran contabilizados entre la población activa. Este problema se extiende a las cifras referidas a la población matri- culada en los diferentes niveles educativos, y al mismo tiempo, aportan una explicación de los bajos niveles formativos de la población.

Concretamente, la población caboverdiana destaca por su bajo nivel de estudios, situándose la tasa de analfabetismo en un 22,5 por ciento (cifras de 2002) y, además existe un menor acceso a los centros educativos para las mujeres, por lo que la tasa de analfabetismo para éstas se cifra en un 30,8 por ciento. Esta situación, unido a la tasa de desempleo existente en el país, cifrada entre un 22 y un 25 por ciento de la población activa (75\%), que se amplía hasta llegar a un 56 por ciento para el caso de las mujeres, suponen un estímulo para el desarrollo del comportamiento emprendedor a pequeña escala, con significativos porcentajes poblacionales que hallan en el autoempleo y las iniciativas empresariales un medio de sustento.

\section{METODOLOGÍA}

Una vez discutidos los factores teóricos que constituyen parte relevante de la estructura social en la que el mercado de empleo se desenvuelve, así como la realidad socioeconómica de la República de Cabo Verde, abordamos el trabajo empírico en el que se combinan las variables de capital humano y psicológicas para explicar las actitudes hacia el empleo en dicho territorio. A tal objeto, en el presente apartado se exponen los aspectos relativos al trabajo de campo desarrollado para recabar la información, se presentan y justifican las variables dependientes y explicativas incluidas en el estudio y, finalmente, se muestra el modelo que sustenta la investigación realizada. 
La influencia de los rasgos psicológicos en las actitudes hacia el empleo

\section{Recogida de información}

La información necesaria para llevar a cabo el presente trabajo de investigación se obtuvo a través de un cuestionario estructurado y redactado en lengua portuguesa al que contestó una muestra representativa de la población caboverdiana. El procedimiento de recogida de información se llevó a cabo mediante una encuesta personal y directa realizada por un encuestador en vías públicas y zonas de confluencia poblacional (e.g., plazas públicas, mercados, eventos sociales públicos, etc.). Para el proceso de recogida de información se seleccionó a tres encuestadores caboverdianos, profesores de secundaria, que hablaban tanto el portugués (lengua oficial de la República) como el criollo (dialecto no escrito de origen portugués mezclado con lenguas africanas, común entre la población de bajos estudios). Esto hizo posible incluir en esta investigación a una muestra verdaderamente representativa de la población caboverdiana puesto que el portugués, al aprenderse en las escuelas, no constituye la lengua de comunicación en la vida cotidiana. Los entrevistadores fueron instruidos sobre los términos y significado de las preguntas incluidas en el cuestionario.

Se definió como universo de investigación a la población residente en Cabo Verde con una edad superior a 15 años, siendo éste el límite habitual de incorporación al mercado laboral. El universo ascendía, por tanto, a 450.489 habitantes en 2002 (población infinita), por lo que admitiendo un nivel de confianza de 95,5 por ciento, el tamaño muestral definido se eleva a 477. A este respecto, la República de Cabo Verde se encuentra dividida en 17 concelhos -forma de división administrativa del territorio nacional- que se encuentran repartidos en 10 islas, dificultando notablemente la recogida de información en el conjunto del archipiélago debido a problemas de accesibilidad y coste económico. Sin embargo, seis de estos 17 concelhos (Tarrafol, Praia, Santa Catarina, Santa Cruz, Säo Miguel, Säo Domingos) están ubicados en una única isla, Santiago, que cuenta con más de la mitad de la población caboverdiana, por lo que optamos por llevar a cabo el trabajo empírico en ellos. Además, estos concelhos constituyen una muestra representativa de la población caboverdiana ya que incluyen tanto población rural como urbana, permitiendo que ambas participen con un peso significativo en el estudio. Específicamente, la población urbana en Cabo Verde se eleva a 248.670 en 2002 , lo que representa el 55,2 por ciento de la población total del país. En la isla de Santiago, lugar en el que se ubican los concelhos seleccionados para el presente estudio, toda la población urbana reside en la ciudad de Praia, perteneciente al municipio del mismo nombre, donde la población urbana representa el 91 por ciento.

En relación al método de muestreo, se ha utilizado el estratificado con afijación proporcional para la isla de Santiago, usando como variables el género, la edad, el nivel de estudios, el concelho y el tipo de municipio (rural/urbano) donde el individuo reside. La muestra final contó con 448 individuos, por lo que se asume un error del 2,3 por ciento, cuya distribución se muestra en la tabla 1.

\section{Variables dependientes}

Para la medida de las actitudes hacia el trabajo, se incluyeron en el cuestionario 
Tabla 1. Distribución de la muestra por estratos en Santiago

\begin{tabular}{|l|l|c|c|}
\hline \multicolumn{2}{|c|}{ Criterios de estratificación } & $\begin{array}{c}\text { Porcentaje en la } \\
\text { población }\end{array}$ & $\begin{array}{c}\text { Porcentaje en la } \\
\text { muestra }\end{array}$ \\
\hline \multirow{3}{*}{ Género } & Mujer & 52,7 & 52,2 \\
\cline { 2 - 4 } & Hombre & 47,3 & 47,8 \\
\hline \multirow{4}{*}{ Edad } & Entre 15 y 64 años & 84,8 & 84,9 \\
\cline { 2 - 4 } & Mayo o igual a 65 años & 15,2 & 15,1 \\
\hline \multirow{5}{*}{ Nivel de estudios } & Analfabeto & 22,6 & 24,0 \\
\cline { 2 - 4 } & Estudios de primaria & 46,4 & 48,3 \\
\cline { 2 - 4 } & Estudios de secundaria & 18,7 & 17,8 \\
\cline { 2 - 4 } & Estudios universitarios & 12,3 & 9,9 \\
\hline \multirow{5}{*}{ Tipo de municipio } & Praia & 45,5 & 43,3 \\
\cline { 2 - 4 } & Tarrafol & 7,7 & 7,4 \\
\cline { 2 - 4 } & Santa Catarina & 20,9 & 22,8 \\
\cline { 2 - 4 } & Santa Cruz & 13,8 & 13,6 \\
\cline { 2 - 4 } & Säo Miguel & 5,4 & 6,7 \\
\cline { 2 - 4 } & Säo Domingos & 6,6 & 6,3 \\
\hline & Urbano & 58,4 & 55,7 \\
\hline
\end{tabular}

preguntas relativas a la actitud hacia el empleo en precario, la actitud hacia la mejora de la formación, la actitud hacia la movilidad y la actitud hacia la emprendeduría. En la tabla 2 se especifican las variables dependientes utilizadas y los autores que sustentan nuestra elección. Estas variables nos permiten medir, en primer lugar, la actitud hacia la aceptación de un empleo en precario a través de la disposición del individuo a trabajar en puestos sin contrato $(\mathrm{Pc})$, con una remuneración inferior a la correspondiente a su categoría profesional (Pr), con una categoría profesional inferior a su nivel de estudios $(\mathrm{Pk})$ y a tiempo parcial $(\mathrm{Pt})$. El estudio de estas actitudes es relevante dado el daño que este tipo de empleo ocasiona en la persona que lo desempeña (Hanisch, 1999; Liem, 1992). Esto es especialmente relevante en un territorio como el caboverdiano donde el limitado nivel de progreso económico va acompa- ñado del desarrollo de una importante economía sumergida.

En segundo lugar, se midió la actitud hacia la mejora de la propia formación a través de la medición de la disposición del individuo a volver a estudiar para mejorar su posición laboral (F). En este sentido, se valoraba tanto la voluntad de formarse para mejorar en el puesto actualmente desempeñado como para acceder a un puesto de trabajo en caso de estar desempleado. La relevancia de la formación para la mejora del capital humano de un territorio y la propia empleabilidad del individuo han sido ampliamente destacados en la literatura (Layard, Nickell y Jackman, 2005; Poirson, 2001), por lo que la actitud de la persona respecto a implicarse en programas formativos no puede quedar excluido de un estudio como el aquí desarrollado, dado los bajos niveles de formación que caracterizan a la población cabo- 
La influencia de los rasgos psicológicos en las actitudes hacia el empleo

Tabla 2. Medida de la actitudes hacia el trabajo

\begin{tabular}{|c|c|c|}
\hline Acrónimo & Variables dependientes & Autores \\
\hline \multicolumn{3}{|c|}{ Actitud hacia el empleo en precario } \\
\hline$(\mathrm{Pc})$ & $\begin{array}{l}\text { Trabajar sin un contrato seguro, escrito e } \\
\text { indefinido }\end{array}$ & \multirow{3}{*}{ Bauder, 2006; Hanisch, 1999} \\
\hline$(\operatorname{Pr})$ & $\begin{array}{l}\text { Aceptar una remuneración inferior a la que } \\
\text { corresponde a su categoría }\end{array}$ & \\
\hline$(\mathrm{Pk})$ & $\begin{array}{l}\text { Aceptar un trabajo de categoría inferior a la de } \\
\text { su formación }\end{array}$ & \\
\hline$(\mathrm{Pt})$ & Trabajar menos de 20 horas semanales & $\begin{array}{l}\text { Bauder, 2006; Felfe et al., 2008; García } \\
\text { Cabrera y García Soto, 2008; Hanisch, } \\
\text { 1999; Yeh et al., } 2007\end{array}$ \\
\hline \multicolumn{3}{|c|}{ Actitud hacia la mejora de la formación } \\
\hline (F) & $\begin{array}{l}\text { Estudiar para conseguir un trabajo o cambiar } \\
\text { de trabajo }\end{array}$ & $\begin{array}{l}\text { Lazarus y Folkman, 1984; Lee y Hsin, } \\
\text { 2004; Sanders y de Grip, 2004 }\end{array}$ \\
\hline \multicolumn{3}{|c|}{ Actitud hacia la movilidad } \\
\hline (Mo) & $\begin{array}{l}\text { Dispuesto a cambiar de oficio para conseguir } \\
\text { un puesto de trabajo mejor o conservar el } \\
\text { actual }\end{array}$ & $\begin{array}{l}\text { Ferri, 2005; Kambourov y Manovskii, } \\
\text { 2002; Moscarini y Thomsson, } 2007\end{array}$ \\
\hline (Mi) & $\begin{array}{l}\text { Dispuesto a cambiar de isla para conseguir un } \\
\text { puesto de trabajo mejor o conservar el actual }\end{array}$ & \multirow{2}{*}{$\begin{array}{l}\text { De Gibson, 2005; Gabriel y Rosenthal, } \\
\text { 1996; Hanisch, 1999; Mcquaid et al., } \\
\text { 2001; Ong y Blumenberg, } 1998\end{array}$} \\
\hline$(\mathrm{Mp})$ & $\begin{array}{l}\text { Dispuesto a cambiar de país para conseguir un } \\
\text { puesto de trabajo mejor o conservar el actual }\end{array}$ & \\
\hline \multicolumn{3}{|c|}{ Actitud hacia la emprendeduría } \\
\hline$(\mathrm{Em})$ & $\begin{array}{l}\text { Decisión de crear una nueva empresa } \\
\text { gestionada por el encuestado }\end{array}$ & $\begin{array}{l}\text { Acs } \text { et al. 2005; Galao Baptista y Vidigal } \\
\text { da Silva, 2004; Minniti et al., 2006; } \\
\text { Reynolds } \text { et al., 2002; Todaro, 1997; } \\
\text { Verheul } \text { et al., } 2006\end{array}$ \\
\hline
\end{tabular}

verdiana. Los esfuerzos económicos y organizativos llevados a cabo por las administraciones públicas para dotar de mayores niveles formativos a la población activa no tendrán los resultados esperados sin el compromiso y/o la voluntad de los individuos por involucrarse en tales iniciativas.

En tercer lugar, la actitud hacia la movilidad se estudia en el presente trabajo a través de la predisposición del encuestado a cambiar de oficio para conseguir un empleo o mejorar el que tiene en la actualidad (Mo), a trasladarse a otra isla (Mi), dado que Cabo Verde es un archipiélago, o incluso fuera del país (Mp). Tanto la reubicación ocupacional como la geográfica constituyen dos aspectos de máxima relevancia debido a la flexibilidad que otorga al mercado de trabajo, facilitando su capacidad para adaptarse a los cambios de tendencia en la actividad económicoindustrial de un territorio (Kambourov y Manovskii, 2002; Moscarini y Thomsson, 2007; Poirson, 2001). Además, y en el nivel individual, la movilidad facilita la empleabilidad de la población activa, así como una mejora de los beneficios salariales y no salariales percibidos como 
resultado de su trabajo. Ambos aspectos justifican la inclusión de la movilidad como actitud hacia el trabajo en la presente investigación desarrollada en un territorio en el que la oferta de empleo se condensa en zonas muy concretas del país y se están desarrollando esfuerzos por impulsar ciertos sectores de actividad como el turístico.

En cuarto lugar, la actitud hacia la emprendeduría se analiza mediante una variable que permite medir la predisposición/decisión del encuestado respecto a crear una nueva empresa gestionada por él mismo. La actividad emprendedora es básica para dinamizar el desarrollo económico de un territorio, siendo especialmente relevante en las economías en transición (Acs et al., 2005; Begley y Tan, 2001; Harper, 1991; Noorderhaven y Tidjani, 2001), además de representar una alternativa de trabajo ante la carencia de empleo por cuenta ajena. De esta forma, dada la condición económica del país, es relevante el estudio de la actitud potencial de la población hacia el autoempleo y puesta en marcha de negocios propios.

Finalmente, destacar que en el cuestionario se incluyeron ocho cuestiones dicotómicas que interrogaban al individuo sobre las mencionadas actitudes hacia el empleo en precario, la mejora de la formación y la movilidad. Estas variables toman el valor 0 cuando el encuestado afirma no estar dispuesto a aceptar la condición definida por la variable y el valor 1 cuando sí lo está. La actitud emprendedora, por su parte, fue medida mediante una variable que valoraba en una escala de cinco posiciones (desde "no lo he valorado, o lo he valorado y he decidido no hacerlo" hasta "lo he valorado y ya la tengo en funcionamiento"), la decisión adoptada por el indi- viduo tras plantearse la creación de un negocio.

\section{Variables explicativas}

Como variables descriptivas del individuo se han incluido las características demográficas y profesionales de los mismos, tales como la edad, el género -variable dicotomía en la mujer toma el valor 0 y el hombre el valor 1-, el nivel de estudios -medido por el número de años completados de escolaridad incluyendo la educación primaria.

Por otra parte, para la medida del locus de control se tuvieron en cuenta las escalas desarrolladas por Levenson (1974), Rotter (1966) y Spector et al. (2001). Acorde a la literatura, hemos construido una medida del locus de control en el trabajo -recomendación de Spector et al. (2001)- que incluye seis ítems de respuesta dicotómica -siguiendo el esquema original de Rotter (1966): (1) conseguir un empleo depende de los conocimientos y capacidades del individuo, (2) conseguir un empleo es una cuestión de suerte, (3) el acceso a un puesto de trabajo depende del lugar donde uno viva; (4) el éxito en el trabajo responde a méritos propios, (5) el éxito en el desempeño profesional es una cuestión de suerte y (6) el éxito en el trabajo depende de otras personas. Dado que carece de sentido aplicar un análisis factorial para escalas con seis ítems de respuesta dicotómica, la dimensión teórica locus de control fue transformada en una variable resumen cuyo valor se calculó mediante la suma de los valores de los ítems que componían dicha escala, siendo posteriormente utilizada en los análisis estadísticos en los que intervenía el locus de control. La idoneidad de la agregación realizada fue constatada 
La influencia de los rasgos psicológicos en las actitudes hacia el empleo

mediante un análisis de correlaciones realizado entre las variables que conforman el constructo. Las correlaciones obtenidas, significativas al nivel del 1 por ciento para todos los pares de variables que integran dicha escala, confirman la relación existente entre los ítemes definidos para la medida $y$, por ende, garantizan que todos ellos analizan un mismo fenómeno -i.e., fiabilidad de la escala.

\section{Modelo de estudio}

Nuestra investigación está basada en la premisa de que la población activa caboverdiana toma decisiones respecto a ampliar su formación, aceptar la movilidad de oficio y/o residencia, aceptar empleos en precario y crear su propia empresa con el fin de acceder o mejorar su empleo. Ahora bien, estas decisiones sobre emprendeduría (Em), formación $(\mathrm{F})$, movilidad -de oficio (Mo), de isla (Mi) y de país (Mp)-, y empleo en precario -sin contrato $(\mathrm{Pc})$, con una remuneración inferior $(\mathrm{Pr})$, con una categoría inferior $(\mathrm{Pk})$ y a tiempo parcial $(\mathrm{Pt})$ - pueden ser estimadas como funciones de variables exógenas que permitan predecir su valor. Estas estimaciones permitirán verificar la influencia de las variables explicativas sobre las variables dependientes analizadas en este estudio.

Con respecto a la emprendeduría, la función lineal múltiple permite, identificar el efecto que las variables independientes género, edad, estudios y locus de control ejercen sobre la variable dependiente (i.e., grado en el que el individuo se involucra con la actividad emprendedora). Para las restantes variables dependientes, de carácter dicotómico, la función lineal logística permite estimar el efecto de las variables explicativas sobre la pertenencia a cada uno de los dos grupos establecidos en cada variable dependiente (e.g., aceptaría versus rechazaría la movilidad de oficio, aceptaría versus rechazaría un empleo a tiempo parcial). Estas aproximaciones lineales de las distintas actitudes hacia el trabajo, en forma resumida, pueden expresarse de la siguiente manera, siendo $E_{i}$ cada una de las variables a explicar:

$$
\begin{gathered}
E_{i}=\beta_{1} \text { GENERO }+\beta_{2} \text { EDAD }+\beta_{3} \text { ESTUDIOS } \\
+\beta_{4} \text { LOCUS }+\beta_{0} ;(i=1, \ldots 9)
\end{gathered}
$$

El análisis de regresión lineal múltiple empleado para la variable dependiente emprendeduría se aplica en esta investigación haciendo uso del método de introducción simultánea, que mediante pasos sucesivos o stepwise determina el conjunto de variables más eficiente. Para la aplicación del análisis de regresión logística binomial, por su parte, se hace uso del método Introduce o Enter para seleccionar el conjunto de variables independientes que más información aporta a cada variable dependiente dicotómica analizada en este trabajo.

\section{RESULTADOS}

Al objeto de ofrecer una adecuada contextualización a las aproximaciones lineales de las actitudes hacia el trabajo estimadas en el presente estudio, procedemos, en primer lugar, y mediante análisis descriptivos y bivariados, a caracterizar a los encuestados en lo que a perfil demográfico y variables psicológicas se refiere, así como a analizar la estructura ocupacional de la muestra y las expectativas hacia el empleo y la actitud emprendedora de la misma. Tras estos análisis preliminares, procedemos a estimar el efecto conjunto de las variables independientes sobre las 
actitudes hacia el trabajo en la República de Cabo Verde.

\section{Perfil del entorno laboral: el contexto de estudio}

La muestra analizada, en clara concordancia con la estructura poblacional de Santiago, cuenta con un 52,2 por ciento de mujeres (véase tabla 1 anterior) y un 68,3 por ciento de individuos con edad inferior a 40 años. Adicionalmente, y en lo que al nivel de estudios se refiere, el 70,6 por ciento tiene una media de menos de 10 años de escolarización, lo que confirma los bajos niveles de formación poblacionales. Merece la pena destacar que el rasgo de personalidad locus de control interno, se sitúa en 2,32 en una escala de tres puntos, lo que indica que, en promedio, los encuestados consideran que el alcance de sus objetivos depende más de sí mismo que de factores externos (véase tabla 3 ).

Por otra parte, y en lo que a tasa de desempleo se refiere, destaca que éste se cifra en torno a un 24 por ciento según el INE de Cabo Verde. Esta elevada tasa de desem- pleo, que constituye un referente sobre el funcionamiento del mercado de trabajo, es común en las economías en desarrollo, justificando la necesidad de establecer políticas públicas paliativas y de desarrollo económico. A este respecto, el interés por reducir el desempleo, va acompañado por la importancia concedida a la reducción de las desigualdades por género, motivo por el cual incorporamos la variable género en los análisis descriptivos relacionados con la estructura ocupacional (véase tabla 4).

En cuanto a ocupación profesional, el peso en la muestra recae en el trabajo artesanal no cualificado o semicualificado $(29,8 \%)$ y en el relacionado con la formación técnica $(16,3 \%)$-e.g., técnico, enfermero o equivalente-, siendo muy minoritarios los puestos de dirección $(3,6 \%)$. Por género, mientras que el empleo masculino se encuentra repartido, en las mujeres la ocupación más común se corresponde con la categoría de trabajos no cualificados $(28,9 \%)$ o trabajos administrativos con formación de carácter general $(16,0 \%)$. Por otra parte, es de destacar el importante papel desempeñado por aquellos que realizan labores no remuneradas $(26,6 \%)$, entre

Tabla 3. Estructura demográfica de la muestra

\begin{tabular}{|c|c|c|c|}
\hline \multicolumn{2}{|c|}{ Estructura demográfica } & Frecuencia & Porcentaje \\
\hline \multirow{4}{*}{$\begin{array}{l}\text { Edad } \\
(n=446)\end{array}$} & Menos de 25 años & 95 & 21,3 \\
\hline & De 25 a 39 años & 210 & 47,0 \\
\hline & De 40 a 59 años & 71 & 15,8 \\
\hline & Mayor o igual a 60 años & 70 & 15,7 \\
\hline \multirow{4}{*}{$\begin{array}{l}\text { Nivel de estudios } \\
(\mathrm{n}=371)\end{array}$} & Menos de 10 años de estudios & 262 & 70,6 \\
\hline & Entre 11 y 13 años & 57 & 15,4 \\
\hline & Entre 14 y 17 años & 45 & 12,1 \\
\hline & Más de 18 años & 7 & 1,9 \\
\hline \multicolumn{2}{|c|}{ Rasgo de personalidad } & Media & DT \\
\hline \multicolumn{2}{|c|}{$\begin{array}{l}\text { Locus interno de control } \\
(\mathrm{n}=422)\end{array}$} & 2,3223 & 0,7553 \\
\hline
\end{tabular}


La influencia de los rasgos psicológicos en las actitudes hacia el empleo

Tabla 4. Estructura ocupacional de la muestra

\begin{tabular}{|c|c|c|c|c|c|}
\hline \multirow{2}{*}{\multicolumn{2}{|c|}{ Estructura ocupacional }} & \multirow{3}{*}{$\begin{array}{l}\text { Frec. } \\
\begin{array}{r}(\%) \\
\\
98 \\
(26,6)\end{array}\end{array}$} & \multicolumn{2}{|c|}{ Género } & \multirow[b]{2}{*}{$X^{2}$} \\
\hline & & & Hombre & Mujer & \\
\hline \multirow{7}{*}{$\begin{array}{l}\text { Profesión } \\
(\mathrm{n}=369)\end{array}$} & No remunerado & & $\begin{array}{r}32 \\
(32,7) \\
(17,6)\end{array}$ & $\begin{array}{r}66 \\
(67,3) \\
(35,3)\end{array}$ & \multirow{7}{*}{$35,438^{\text {水水* }}$} \\
\hline & Trabajador no cualificado & $\begin{array}{r}110 \\
(29,8)\end{array}$ & $\begin{array}{r}56 \\
(50,9) \\
(30,8)\end{array}$ & $\begin{array}{r}54 \\
(49,1) \\
(28,9)\end{array}$ & \\
\hline & $\begin{array}{l}\text { Administrativo o secretario/a } \\
\text { con formación de carácter } \\
\text { general }\end{array}$ & $\begin{array}{r}48 \\
(13,0)\end{array}$ & $\begin{array}{r}18 \\
(37,5) \\
(9,9)\end{array}$ & $\begin{array}{r}30 \\
(62,5) \\
(16,0)\end{array}$ & \\
\hline & Formación técnica & $\begin{array}{r}60 \\
(16,3)\end{array}$ & $\begin{array}{r}47 \\
(78,3) \\
(25,8)\end{array}$ & $\begin{array}{r}13 \\
(21,7) \\
(7,0)\end{array}$ & \\
\hline & $\begin{array}{l}\text { Profesional con formación } \\
\text { académica o equivalente }\end{array}$ & $\begin{array}{r}40 \\
(10,8)\end{array}$ & $\begin{array}{r}22 \\
(55,0) \\
(12,1) \\
\end{array}$ & $\begin{array}{r}18 \\
(45,0) \\
(9,6) \\
\end{array}$ & \\
\hline & $\begin{array}{l}\text { Directivo con uno o más } \\
\text { subordinados }\end{array}$ & $\begin{array}{r}12 \\
(3,3)\end{array}$ & $\begin{array}{r}6 \\
(50,0) \\
(3,3)\end{array}$ & $\begin{array}{r}6 \\
(50,0) \\
(3,2) \\
\end{array}$ & \\
\hline & Alta dirección & $\begin{array}{r}1 \\
(0,3)\end{array}$ & $\begin{array}{r}1 \\
(100,0) \\
(0,5)\end{array}$ & $\begin{array}{r}0 \\
(0,0) \\
(0,0)\end{array}$ & \\
\hline \multirow{2}{*}{$\begin{array}{l}\text { Igualdad profesional } \\
\text { hombre/mujer } \\
(n=429)\end{array}$} & Se percibe igualdad & $\begin{array}{r}163 \\
(38,0)\end{array}$ & $\begin{array}{r}82 \\
(50,3) \\
(40,4)\end{array}$ & $\begin{array}{r}81 \\
(49,7) \\
(35,8)\end{array}$ & \multirow{2}{*}{0,941} \\
\hline & Se percibe desigualdad & $\begin{array}{r}266 \\
(62,0)\end{array}$ & $\begin{array}{r}121 \\
(45,5) \\
(59,6)\end{array}$ & $\begin{array}{r}145 \\
(54,5) \\
(64,2)\end{array}$ & \\
\hline
\end{tabular}

***** $p<0,001$

los que predomina el colectivo de mujeres (67,3\% frente al $32,7 \%$ de los hombres). Tómese en consideración que, sobre todo en las zonas rurales, la mujer contribuye con su trabajo al desarrollo de la actividad profesional del marido, sin que perciba un salario por ello. Finalmente, debe destacarse que los encuestados mayoritariamente perciben la existencia de desigualdad por género en el mercado de trabajo $(62 \%)$. Tales desigualdades afectan a las oportunidades de empleo, entre otros aspectos, creando discriminación contra la mujer (véase tabla 4 anterior).
Adicionalmente, se han analizado las actitudes hacia el trabajo de los encuestados (e.g. disponibilidad para trabajar en precario, movilidad, mejora de formación, predisposición al autoempleo, etc.). En primer lugar, y con respecto a la precariedad laboral, es de destacar que ésta es asumida por la mayoría de los encuestados, que muestran una actitud favorable a aceptar este tipo de condiciones. Concretamente, un 51,5 por ciento manifiesta estar dispuesto a aceptar empleos sin contrato, un 79,3 por ciento a percibir una remuneración inferior a la que le corres- 
ponde por su categoría y un 83,4 por ciento a aceptar un trabajo con una categoría inferior a la que le pertenece. Por su parte, el 87,3 por ciento de los encuestados afirma estar dispuesto a trabajar menos de 20 horas semanales si estuviese desempleado -empleo a tiempo parcial-. Por otra parte, una mayoría de los encuestados manifiestan estar dispuestos a volver a estudiar con el propósito de mejorar su formación para conseguir un puesto de trabajo o mejorar el actual $(79,3 \%)$. En lo que a la actitud hacia la movilidad se refiere, los encuestados muestran una alta disposición hacia la misma. Concretamente, el 85,6 por ciento declara que estaría dispuesto a cambiar de oficio para conseguir un puesto de trabajo mejor. Asimismo, el 73,8 por ciento cambiaría de isla y el 75,8 por ciento de país para conseguir esa mejor situación laboral (véase tabla 5).

\section{Tabla 5. Actitudes hacia el trabajo}

\begin{tabular}{|c|c|c|c|}
\hline \multicolumn{2}{|c|}{ Actitudes hacia el trabajo } & Frecuencias & Porcentaje \\
\hline \multicolumn{4}{|l|}{ Actitud hacia el empleo en precario } \\
\hline \multirow{2}{*}{$\begin{array}{l}\text { Trabajar sin un contrato seguro, escrito e } \\
\text { indefinido } \\
(n=445)\end{array}$} & No & 216 & 48,5 \\
\hline & Sí & 229 & 51,5 \\
\hline \multirow{2}{*}{$\begin{array}{l}\text { Aceptar una remuneración inferior a la que } \\
\text { corresponde a su categoría } \\
(n=444)\end{array}$} & No & 92 & 20,7 \\
\hline & Sí & 352 & 79,3 \\
\hline \multirow{2}{*}{$\begin{array}{l}\text { Aceptar un trabajo de categoría inferior a la } \\
\text { de su formación } \\
(\mathrm{n}=445)\end{array}$} & No & 74 & 16,6 \\
\hline & Sí & 371 & 83,4 \\
\hline \multirow{2}{*}{$\begin{array}{l}\text { Trabajar menos de } 20 \text { horas semanales, si } \\
\text { estoy desempleado } \\
(n=441)\end{array}$} & No & 56 & 12,7 \\
\hline & Sí & 385 & 87,3 \\
\hline \multicolumn{4}{|l|}{ Actitudes hacia la mejora de la formación } \\
\hline \multirow{2}{*}{$\begin{array}{l}\text { Estudiar para conseguir un trabajo o } \\
\text { cambiar de trabajo } \\
(\mathrm{n}=439)\end{array}$} & No & 91 & 20,7 \\
\hline & Sí & 348 & 79,3 \\
\hline \multicolumn{4}{|l|}{ Actitudes hacia la movilidad } \\
\hline \multirow{2}{*}{$\begin{array}{l}\text { Dispuesto a cambiar de oficio para } \\
\text { conseguir un puesto de trabajo mejor o } \\
\text { conservar el actual } \\
(\mathrm{n}=438)\end{array}$} & No & 63 & 14,4 \\
\hline & Sí & 375 & 85,6 \\
\hline \multirow{2}{*}{$\begin{array}{l}\text { Dispuesto a cambiar de isla para conseguir } \\
\text { un puesto de trabajo mejor o conservar el } \\
\text { actual } \\
(\mathrm{n}=447)\end{array}$} & No & 117 & 26,2 \\
\hline & Sí & 330 & 73,8 \\
\hline \multirow{2}{*}{$\begin{array}{l}\text { Dispuesto a cambiar de país para conseguir } \\
\text { un puesto de trabajo mejor o conservar el } \\
\text { actual } \\
(\mathrm{n}=447)\end{array}$} & No & 108 & 24,2 \\
\hline & Sí & 339 & 75,8 \\
\hline \multicolumn{2}{|l|}{ Actitud hacia la emprendeduría } & Media & DT \\
\hline \multicolumn{2}{|l|}{$\begin{array}{l}\text { Comportamiento emprendedor } \\
(\mathrm{n}=440)\end{array}$} & 1,6159 & 0,6851 \\
\hline
\end{tabular}


La influencia de los rasgos psicológicos en las actitudes hacia el empleo

Por otra parte, los encuestados muestran una inclinación hacia el comportamiento emprendedor, al alcanzar éste un valor promedio de 1,6 -min. 1 y máx. 3(véase tabla 5 anterior). Tómese en consideración que el valor 1 se corresponde con el mínimo de la escala, donde el individuo rechaza la idea de crear su propio negocio, situándose en este valor sólo el 50 por ciento de la muestra. Los resultados revelan una considerable inclinación hacia el autoempleo, lo que confirma que el desempleo es una fuerza conductora de la emprendeduría a pequeña escala.

El análisis realizado en este epígrafe corrobora la existencia de un elevado nivel de desempleo en la República de Cabo Verde. Éste, se ve agravado por el perfil demográfico y de capital humano de la población caboverdiana: (1) bajo nivel de estudios, (2) ocupación preferente en puestos de baja cualificación y (3) edad joven a la que se accede al mercado laboral. A ello se une la existencia de discriminación laboral por razón de género. Ante esta situación general, adquiere una gran relevancia los valores del locus de control interno, pues aportan un valor potencial adicional al capital humano caboverdiano. Además, un grupo significativo de la muestra está comprometida con aquellas condiciones que mejoran su empleabilidad, así como con la emprendeduría.

\section{Factores determinantes de las actitudes hacia el trabajo}

En la tabla 6a y 6 b se recogen los resultados de las ocho regresiones logísticas estimadas para el análisis de las actitudes hacia el trabajo y los referidos a la regresión múltiple para la actitud hacia la emprendeduría. Con respecto a las prime- ras, las pruebas Chi-cuadrado realizadas muestran la bondad de ajuste de los modelos estimados. Por su parte, esta bondad de ajuste también se observa para la regresión múltiple a través del estadístico $F$ y la prueba Durbin-Watson. Adicionalmente, los coeficientes beta estimados y testados a partir del estadístico de Wald para la regresión logística y $t$ de Student para la regresión múltiple, nos permiten hallar los efectos significativos de las variables independientes sobre las actitudes hacia el trabajo y la emprendeduría. En cuanto a los estadísticos $\mathrm{R}^{2}$, cabe destacar los elevados niveles que estos muestran para las regresiones que estiman las actitudes de los encuestados hacia ampliar su formación $(39,3 \%)$, aceptar la movilidad (oscila entre $29,6 \%$ para la movilidad de oficio y el $38,5 \%$ para la movilidad de isla) y aceptar empleos en precario (varía entre 18,1\% y $27,9 \%$, dependiendo de la forma de precariedad estimada). Sin embargo, y en lo que se refiere a la actitud hacia la creación de empresas, el estadístico muestra un exiguo 3,6 por ciento lo que indica que existe un conjunto de variables no incorporado en este estudio que contribuyen a explicar dicha actitud hacia la emprendeduría. Ahora bien, dado que esta investigación no está interesada en conocer todos los factores que explican la actitud emprendedora sino en determinar la capacidad explicativa del locus de control en relación a las distintas actitudes del individuo hacia el trabajo, este $\mathrm{R}^{2}$ bajo no afecta a los resultados buscados en la presente investigación.

Con respecto al género, no se observa una influencia estadísticamente significativa sobre la disposición a aceptar condiciones laborales precarias tales como la aceptación de empleos sin contrato $(\mathrm{Pc})$, con una remuneración inferior a la correspon- 
Tabla 6a. Análisis de regresión de las actitudes hacia el trabajo del individuo

\begin{tabular}{|c|c|c|c|c|c|}
\hline \multicolumn{6}{|c|}{ Regresión logística binaria } \\
\hline & $\begin{array}{c}\text { (1) } \\
\text { Aceptar } \\
\text { empleos sin } \\
\text { contrato } \\
\text { (Pc) } \\
\end{array}$ & $\begin{array}{c}(2) \\
\text { Aceptar } \\
\text { empleos con } \\
\text { una } \\
\text { remuneración } \\
\text { inferior } \\
\text { (Pr) } \\
\end{array}$ & $\begin{array}{c}\text { (3) } \\
\text { Aceptar } \\
\text { empleos con } \\
\text { una categoría } \\
\text { inferior } \\
(\mathbf{P k}) \\
\end{array}$ & $\begin{array}{c}\text { (4) } \\
\text { Aceptar } \\
\text { empleos a } \\
\text { tiempo parcial } \\
\text { (Pt) }\end{array}$ & $\begin{array}{c}\text { (5) } \\
\text { Ampliar } \\
\text { la formación }\end{array}$ \\
\hline & Beta Wald & Beta Wald & Beta Wald & Beta Wald & Beta Wald \\
\hline Constante & $\mathbf{2}, \mathbf{3 3 6} 26,803$ *水氺 & $\mathbf{4 , 6 0 3} 52,433$ *水水 & $\mathbf{6 , 0 2 0} 60,018$ *水*1 & $\mathbf{5 , 9 8 7 5 2 , 1 7 7}$ 水水米 & $\mathbf{4 , 0 2 5} 44,708$ 水水米 \\
\hline Género & $-0,293 \quad 1,832$ & $-0,064 \quad 0,057$ & $-0,134 \quad 0,192$ & $-0,104 \quad 0,096$ & $\mathbf{- 0 , 5 2 0} 3,097^{\dagger}$ \\
\hline Edad & $\mathbf{- 0 , 1 1 5} 6,355 *$ & $\mathbf{- 0 , 2 7 5} 24,690$ *水水 & 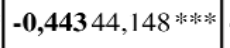 & $\mathbf{- 0 , 4 6 0} 39,478$ 水水淑 & 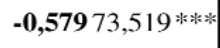 \\
\hline Estudios & $\mathbf{- 0 , 5 3 1} 35,298 * *$ & $\mathbf{- 0 , 2 3 9} 18,155^{* * * *}$ & $-\mathbf{0 , 2 7 9} 20,209 * * *$ & $\mathbf{- 0 , 2 1 2} 9,377^{* *}$ & $\mathbf{0 , 1 7 5} 3,556^{\dagger}$ \\
\hline Locus & $\mathbf{- 0 , 3 2 3} 4,848$ * & $\mathbf{- 0 , 5 4 9} 7,524 * *$ & $\mathbf{- 0 , 5 3 7} 5,384 *$ & $-0,390 \quad 2,497$ & $0,226 \quad 1,389$ \\
\hline$X^{2}$ & $79,987^{* * *} *$ & $49,776^{* * *}$ & $72,524 * * * *$ & $55,009^{* * * *}$ & $117,632^{* * * *}$ \\
\hline $\begin{array}{c}\mathrm{R}^{2} \\
\text { Negelkerke }\end{array}$ & $23,5 \%$ & $18,1 \%$ & $27,9 \%$ & $24,4 \%$ & $39,3 \%$ \\
\hline
\end{tabular}

${ }^{\dagger} p<0,1, * p<0,05, * * p<0,01, * * * p<0,001$.

Tabla 6b. Análisis de regresión de las actitudes hacia el trabajo del individuo

\begin{tabular}{|c|c|c|c|}
\hline \multicolumn{3}{|c|}{ Regresión logística binaria } & \multirow{2}{*}{$\begin{array}{c}\text { Regresión múltiple } \\
\text { (9) } \\
\text { Emprendeduría }\end{array}$} \\
\hline $\begin{array}{c}\text { (6) } \\
\text { Aceptar la } \\
\text { movilidad de } \\
\text { oficio }\end{array}$ & $\begin{array}{c}\text { (7) } \\
\text { Aceptar la } \\
\text { movilidad de } \\
\text { isla }\end{array}$ & $\begin{array}{c}(8) \\
\text { Aceptar la } \\
\text { movilidad de } \\
\text { país }\end{array}$ & \\
\hline (Mo) & (Mi) & (Mp) & $(\mathbf{E m})$ \\
\hline Beta Wald & Beta Wald & Beta Wald & Beta \\
\hline $7,12061,255$ * * * * & $3,44640,672$ 水水米 & $4,67659,530$ *水水 & $\mathbf{1 , 5 9 7} 11,643$ **⿰㇇⿰亅⿱丿丶丶 \\
\hline$-0,043 \quad 0,018$ & $-0,139 \quad 0,266$ & $0,291 \quad 1,111$ & $\mathbf{- 0 , 2 2 \longdiv { - 3 , 2 5 }} 6 * * *$ \\
\hline $\mathbf{- 0 , 4 5 8} 42,932$ 水水米 & $\mathbf{- 0 , 5 7 0} 82,108$ 水水* & 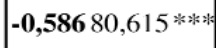 & $\mathbf{0 , 0 4 0} 2,769 * *$ \\
\hline$-\mathbf{0 , 2 2 4} 11,758 * * *$ & $\mathbf{0 , 1 3 0} 2,776^{\dagger}$ & $\mathbf{- 0 , 1 7 4} 7,800 * *$ & 0,021 \\
\hline $\mathbf{- 0 , 9 4 0} 12,248$ 水水深 & $0,211 \quad 1,431$ & $-0,101 \quad 0,290$ & $-0,039 \quad-0,838$ \\
\hline $72,885^{* * *}$ & $125,936^{* * * *}$ & $114,486^{* * * *}$ & \multirow{2}{*}{$\begin{array}{c}F=4,767 * * * \\
\mathrm{R}^{2} \text { ajustado=3,6\% } \\
\text { Durban-Watson=1,916 }\end{array}$} \\
\hline $29,6 \%$ & $38,5 \%$ & $36,2 \%$ & \\
\hline
\end{tabular}

${ }^{\dagger} p<0,1, * p<0,05, * * p<0,01, * * * p<0,001$. 
diente por categoría profesional (Pr), con una categoría profesional inferior al nivel de estudios $(\mathrm{Pk})$ o en empleos a tiempo parcial $(\mathrm{Pt})$, así como en las decisiones de aceptar la movilidad tanto de oficio (Mo) como de isla (Mi) o país (Mp). Sin embargo, esta variable sí ejerce una influencia significativa sobre la decisión de ampliar la formación para encontrar un empleo (F), demostrando que las mujeres están, en promedio, más dispuestas a mejorar su formación con este fin. Por su parte, la variable género también afecta al comportamiento emprendedor del individuo, siendo mayor entre el colectivo de las mujeres que en el de los varones, lo que es contradictorio con buena parte de la literatura publicada que asocia el comportamiento emprendedor a individuos de sexo masculino (Begley y Tan, 2001; Cowling y Taylor, 2001; Ardichvili y Gasparishvili, 2003; Acs et al., 2005). Estos resultados revelan que, en nuestra muestra, las mujeres contemplan la opción de autoempleo y la ampliación de su formación por encima de lo que lo hacen los hombres.

La edad del individuo, por otra parte, muestra tener una influencia estadísticamente significativa en todas las regresiones. Concretamente, cuanto mayor es la edad del individuo menor será su predisposición a aceptar situaciones de precariedad en el empleo -sin contrato (Pc), con remuneración inferior a la categoría profesional (Pr), con categoría profesional inferior al nivel de estudios $(\mathrm{Pk})$ o en empleos a tiempo parcial $(\mathrm{Pt})-$, menor será también su negativa a ampliar su formación para acceder o mejorar el puesto de trabajo actual (F) y a aceptar un cambio de oficio (Mo), isla (Mi) o país (Mp). Estos resultados permiten deducir tres tendencias importantes en el mercado de trabajo caboverdiano: (1) la mayor predisposición de los más jóvenes a aceptar situaciones "injustas" en lo que a precariedad se refiere para acceder al mercado de trabajo, debido probablemente a su mayor dificultad para encontrar un empleo (discriminación por edad); (2) la mayor inmovilidad e incapacidad de adaptación de los individuos de más edad, que se muestran reticentes a ampliar su formación o aceptar la movilidad ocupacional y/o territorial para acceder o mejorar su empleo; y (3) la relevancia del colectivo de los más jóvenes en el mercado de trabajo caboverdiano, pues son los que aportan mayor predisposición a asumir condiciones que generan dinamismo en el mismo. Estos resultados, sin embargo, no se extienden al comportamiento emprendedor, mostrando la regresión estimada un efecto significativo y positivo de la edad sobre esta variable, de forma que son los individuos de mayor edad los que, en mayor medida, cuentan con su propio negocio. Esto podría considerarse que contrasta con la literatura que asocia el comportamiento emprendedor a individuos de no elevada edad (Acs et al., 2005; Ardichvili y Gasparishvili, 2003). Sin embargo, dado que no estamos analizando la edad del individuo en el momento en que decidió crear su empresa, sino sus características actuales, tal contradicción no existe.

El nivel de estudios también muestra una influencia estadísticamente significativa en la predisposición a aceptar un empleo en precario y hacia la movilidad, no ocurriendo así con respecto a la decisión de crear un negocio propio. Así, y en primer lugar, las regresiones logísticas estimadas muestran cómo cuanto mayor es el nivel de estudios del individuo menor será su inclinación a aceptar un puesto de trabajo sin contrato $(\mathrm{Pc})$, con remuneración inferior a la categoría profesional 
(Pr), con categoría profesional inferior al nivel de estudios (Pk) o a tiempo parcial (Pt). En segundo lugar, el nivel educativo también tiene una influencia directa y negativa en la actitud hacia la movilidad de oficio (Mo) y de país (Mp), pero muestra un efecto positivo en la movilidad de isla. Todo ello se explica por la mayor empleabilidad de los mismos por su valor diferencial en un mercado de trabajo caracterizado por los bajos niveles educativos de la población activa. Adicionalmente, el nivel educativo muestra una incidencia directa y positiva en la decisión de volver a estudiar para acceder o mejorar un empleo, lo que demuestra el valor que los individuos más formados conceden al conocimiento como mecanismo de mejora de las condiciones de trabajo. Finalmente, y contrariamente a lo establecido en la literatura que asocia el comportamiento emprendedor a individuos de mayor nivel de estudios (Acs et al., 2005; Ardichvili y Gasparishvili, 2003), la regresión múltiple (Em) no confirma esta influencia. La inclinación de la población hacia el autoempleo cuando las opciones de trabajo por cuenta ajena son escasas, tal y como es común en países en vías de desarrollo (Acs et al., 2005; Reynolds et al., 2002), podría justificar que el nivel educativo del individuo no explique el comportamiento emprendedor en la República de Cabo Verde.

Asimismo, y en lo que al locus de control se refiere, los análisis estadísticos realizados muestran cómo los individuos con mayor control interno como rasgo de personalidad, están menos dispuestos a aceptar las condiciones precarias de trabajo. Estos resultados se obtienen en lo que se refiere a la aceptación de un empleo sin contrato $(\mathrm{Pc})$, con remuneración inferior a la correspondiente por categoría profesio- nal (Pr) y con categoría profesional inferior al nivel de estudios (Pk). Este rechazo se justifica en la mayor confianza de los individuos de locus interno en su capacidad personal para lograr un puesto de trabajo acorde a su preparación y perfil. Adicionalmente, el locus de control también influye en la predisposición a la movilidad del individuo ya que cuanto mayor es el locus interno menor es la predisposición a cambiar de oficio para encontrar un empleo (Mo). Finalmente, y aunque el locus de control interno ha sido teóricamente asociado a un mayor comportamiento emprendedor (McClelland, 1961; Mueller y Thomas, 2000) -aunque con resultados contradictorios en la investigación empírica-, en la regresión estimativa del comportamiento emprendedor (Em) hallada en el presente trabajo, esta influencia no se encuentra presente. Ello podría ser debido nuevamente a la importancia de la "emprendeduría por necesidad" en Cabo Verde.

Atendiendo a los resultados obtenidos, destaca que son los individuos más jóvenes, de menor nivel educativo y de menor locus interno de control los que están en mayor medida dispuestos a aceptar condiciones de trabajo en precario para acceder a un empleo. Esto puede sólo significar que el estrés causado por el desempleo condiciona de facto una actitud favorable hacia el infraempleo. Estos resultados sugieren la necesidad de que en la República de Cabo Verde se adopten las medidas necesarias para regularizar los puestos de trabajo ofrecidos en el mercado laboral, de forma que la aceptación de puestos irregulares sin contrato no se convierta en el elemento diferenciador del capital humano que condicione su empleabilidad. Además, los coeficientes beta estandarizados demuestran que, en los modelo logísti- 
cos, la edad y el locus de control son, con carácter general, las variables explicativas con mayor influencia sobre las actitudes hacia el trabajo, lo que corrobora la importancia del rasgo psicológico locus de control como variable a ser considerada en los estudios referidos al mercado de trabajo. Con respecto a la emprendeduría, es el género la que tiene una mayor influencia.

\section{CONCLUSIONES}

Esta investigación, llevada a cabo con el propósito de estudiar la relación de los perfiles demográficos y psicológicos con las actitudes hacia el trabajo en la República de Cabo Verde, se ha construido sobre las variables comúnmente ligadas al estudio del capital humano (género, edad y nivel de estudios). Además, y bajo una perspectiva integradora, se ha incorporado el estudio del locus de control como rasgo de personalidad del individuo, pues constituye un activo valioso con capacidad para explicar su comportamiento y resultados en el mercado de trabajo. Entre las actitudes hacia el empleo se han considerado tanto aquellas que afectan a la empleabilidad del trabajador -disposición a volver a estudiar para mejorar la posición laboral, a trabajar en puestos en precario sin contrato, con una remuneración inferior a la correspondiente a su categoría profesional, con una categoría profesional inferior a su nivel de estudios y a tiempo parcial, a cambiar de oficio para conseguir un empleo o mejorar el que tiene en la actualidad, a trasladarse a otra isla o incluso fuera del país- como a su disposición al autoempleo o al desarrollo de iniciativas empresariales.

La información estadística recopilada confirma la existencia de un elevado nivel de desempleo, como es habitual en economías en transición, que afecta con mayor virulencia a las mujeres. Éste, se ve agravado por el perfil demográfico y de capital humano de la población caboverdiana: bajo nivel de estudios, ocupación preferente en puestos de baja cualificación y edad joven a la que se accede al mercado laboral (con limitada experiencia previa). A ello se une la existencia de discriminación laboral por razón de género que afecta negativamente a la mujer y que no sólo se concluye en los datos referidos a las tasas de desempleo sino que también afecta al tipo de ocupación: la mujer se emplea mayoritariamente en puestos administrativos, o para los que no se requiere cualificación, estando reservados los directivos, profesionales y técnicos al varón. Esta discriminación es, además, percibida y manifestada tanto por los varones como por las mujeres participantes en el estudio.

Ante esta situación general negativa, el estudio de los rasgos psicológicos de la población adquiere una gran relevancia pues puede contribuir a esclarecer el valor potencial del capital humano existente en dicho país, dado que tales rasgos inciden en las actitudes hacia el trabajo y, por ende, en el desarrollo económico del país. Específicamente, los análisis realizados muestran que la población caboverdiana se inclina hacia la convicción personal de que los acontecimientos que se producen en la vida diaria son el resultado de sus habilidades y de sus esfuerzos. Además, estadísticamente se observa que los individuos con elevado locus interno responden a un perfil diferenciado del resto de la población que afecta a su empleabilidad. Estos individuos rechazan en mayor medida los puestos en economía sumergida, donde los contratos en precario son comu- 
nes y, al mismo tiempo, también muestran baja disposición a cambiar de oficio. Estos resultados indican que el rasgo de personalidad locus de control se asocia a la confianza del individuo en sus capacidades y habilidades para afrontar el reto que el mercado laboral aporta en su país, por lo que no se ven obligados a participar en el infraempleo.

Por otra parte, y atendiendo a las variables demográficas contempladas en el estudio, los resultados muestran una actitud diferenciada hacia las actitudes hacia el trabajo y la emprendeduría en razón del género, la edad y el nivel de estudios. En lo que a género se refiere, destaca una mayor actitud activa hacia el empleo por parte de la mujer caboverdiana, en un intento de superación de las desigualdades. Concretamente, la mujer, a diferencia del hombre, muestra mayor disposición a incrementar su capacitación para conseguir un puesto de trabajo, lo que avala su mayor compromiso con la lucha por superar el desempleo. Estos resultados obtenidos en la República de Cabo Verde son concordantes con los hallados por Schafgans (2000) en Malasia, donde la fuerza de trabajo femenina creció más rápidamente que la masculina debido a la mejora en los niveles educativos de la mujer y a su actitud más favorable hacia el empleo. Por consiguiente, Cabo Verde no constituye un caso atípico en lo que al comportamiento de la mujer se refiere en este contexto laboral en una economía en desarrollo.

Entre las actitudes hacia el trabajo, en el presente estudio también se aborda la actitud de la mujer hacia la emprendeduría. Nuestra investigación ha hallado una disposición emprendedora superior en la mujer que en el varón caboverdiano, contrariamente a lo establecido en la literatura que sostiene que el emprendedor es generalmente varón. Dos argumentos pueden explicar estos resultados. Por una parte, el mayor efecto que el desempleo ejerce en el comportamiento emprendedor de las féminas que, según Verheul et al. (2006), no es extensivo a los varones y, además, se acentúa en economías en transición. A ello habría que unir el elevado desempleo y discriminación laboral de la mujer en Cabo Verde. Por otra, y si consideramos las especificidades sociales de este país, los resultados también se justifican cómodamente, ya que la sociedad caboverdiana confiere a la mujer una responsabilidad casi sin límites con relación al sustento y manutención de la familia, siendo prácticamente la única responsable de la educación de los hijos. Ello obedece a que la poligamia masculina constituye una práctica habitual e institucionalizada, como ya ha sido mencionado. Ahora bien, para Cowling y Hayward (2000), el paso de la mujer del desempleo al autoempleo obedece a su deseo de mantener el sustento familiar incluso aunque viva en pareja, si su compañero pierde el empleo.

Con respecto a la edad, este trabajo revela un claro compromiso de los más jóvenes con las condiciones que afectan a su empleabilidad, al mostrar un actitud más favorable a ampliar su formación para acceder al mercado de trabajo, a emplearse en puestos a tiempo parcial, a aceptar la movilidad ocupacional y/o territorial e, incluso, a aceptar el infraempleo y la precariedad. Todo ello viene a corroborar, en primer lugar, el empuje positivo que los más jóvenes aportan al mercado laboral caboverdiano; en segundo lugar, que la anteriormente citada discriminación por género se extiende a la discriminación por razón de edad, lo que se ve agudizado por los bajos niveles de formación y experien- 
cia laboral del colectivo de los más jóvenes. Por otra parte, son los individuos de más edad los que muestran un mayor comportamiento emprendedor, al estar en la actualidad dirigiendo su negocio. De esta forma, la menor predisposición de estos individuos a tomar decisiones que favorezcan su empleabilidad (ampliar su formación, aceptar la movilidad ocupacional y/o territorial) puede verse moderada por su mayor compromiso con el comportamiento emprendedor o el autoempleo, aportando otro componente de importancia para el dinamismo del mercado de trabajo caboverdiano.

El nivel de estudios también demuestra ser para la República de Cabo Verde una variable influyente en las actitudes adoptadas hacia la empleabilidad por cuenta ajena pero no hacia la emprendeduría. Concretamente, cuanto mayor es el nivel de estudios del individuo menor será su inclinación a aceptar el infraempleo, la movilidad ocupacional o la emigración hacia el extranjero. Lógicamente, los individuos de mayor nivel de estudios y grado de especialización rechazarán en mayor medida el cambio de oficio, debido al coste de oportunidad y a la pérdida de competencia que ello le ocasionaría. Asimismo, es relevante el hecho de que los individuos de mayor nivel de estudios rechacen el cambio de país, pero no de isla, para conseguir o mejorar un empleo, lo que puede venir justificado por su mayor facilidad para encontrar empleo en un contexto de bajo nivel educativo. Esta actitud de la población de mayor estudio, por otra parte, impide la descapitalización intelectual de la República de Cabo Verde. Finalmente, los individuos de mayor nivel de estudios muestran, además, un mayor compromiso con la formación, al estar en mayor medida dispuestos a continuar ampliando la misma. Esta sensibilidad refleja el efecto expansivo que la inversión en formación podría generar.

Sin embargo, el nivel de estudios no muestra incidencia sobre la actitud emprendedora, a pesar de ser un factor que condiciona la capacidad del individuo para percibir las distintas oportunidades empresariales existentes en el entorno (Basu, 1998; Mitchell, Smith, Sewright y Morse, 2000), así como por facultarle en el acceso a las diferentes instancias, procedimientos y agentes necesarios para la creación y puesta en marcha de la empresa (Arenius y de Clercq, 2005; Mitchell et al., 2000). Ahora bien, dado que las iniciativas empresariales de los caboverdianos, en muchos casos, se corresponden con la emprendeduría informal, esto es, pequeños negocios no constituidos formalmente, el efecto del nivel educativo se minimiza. A ello se une, por otra parte, el factor “emprendeduría por necesidad", según el cual es la imposibilidad de encontrar un empleo por cuenta ajena, más que otro factor, lo que justifica la decisión de crear un negocio propio.

La presente investigación tiene implicaciones prácticas que afectan a la programación de las políticas de inserciónempleo. En primer lugar, la importancia de elevar los niveles de estudios de la población caboverdiana debido a su incidencia en la empleabilidad de los mismos queda, sin lugar a dudas, ratificada en el presente trabajo. De esta forma, constituye un gran reto para las administraciones públicas caboverdianas superar el déficit formativo que caracteriza a su población activa. En segundo lugar, los resultados de este estudio pueden orientar, de forma más precisa, las actividades formativas dirigidas a la población activa, así como 
a los contenidos educativos incluidos desde la formación primaria y secundaria (Groves, 2005). Concretamente, y dado el efecto beneficioso que el locus de control interno tiene sobre las variables explicativas de la actitud hacia el trabajo del individuo, los programas de formación deberían incluir la docencia en actitudes conductuales que refuercen la confianza en la propia capacidad del individuo y la conexión entre los logros personales alcanzados en el mercado de trabajo y el esfuerzo/sacrificio propio. Asimismo, podrían estimular en el individuo la confianza en su capacidad para actuar en contra de las amenazas identificadas en el entorno más que su dependencia absoluta del mismo. La mejora de estas actitudes no sólo pueden reducir los costes económicos y sociales del desempleo, sino que también permiten dinamizar el mercado de trabajo, incrementar la productividad $\mathrm{y}$, por ende, el crecimiento económico del país.

En segundo lugar, y a pesar de los esfuerzos llevados a cabo en la República de Cabo Verde por reducir la desigualdad hacia la mujer, los resultados de este trabajo confirman la necesidad de intensificar las actuales políticas a favor de la igualdad de oportunidades, que además deben extenderse a la discriminación por razón de edad, ampliando la adopción de medidas de discriminación positiva que garanticen la plena igualdad de derechos de la mujer y de los más jóvenes en la práctica. Adicionalmente, el desarrollo de acciones educativas dirigidas a la sociedad en aras a estimular una conciencia colectiva que respete la igualdad, parece también conveniente.

En tercer lugar, los resultados sugieren la necesidad por parte de las instituciones públicas de la República de Cabo Verde de adoptar las medidas tanto de carácter legislativo como de estímulo de la responsabilidad social de los empresarios con el propósito de combatir el infraempleo y regularizar los puestos de trabajo actualmente en precario. En este sentido, es de máxima importancia que la predisposición a aceptar puestos de trabajo irregulares no se convierta en el elemento diferenciador del capital humano caboverdiano.

Finalmente, conviene destacar las limitaciones del presente trabajo de investigación. Entre ellas mencionamos, en primer lugar, la no inclusión de otras variables explicativas que miden el capital humano, así como otros rasgos de personalidad del individuo. En este sentido, debemos destacar que la necesidad de construir un cuestionario de una dimensión adecuada, que promoviese la cooperación de la población caboverdiana en el proceso de recogida de datos, hizo necesario centrar este estudio en pocas variables, cuyo análisis fuese factible en un contexto en el que predomina la población con bajos estudios. Por otra parte, destacamos también la obtención de un coeficiente $\mathrm{R}^{2}$ especialmente bajo para la regresión que estimaba la actitud hacia la creación de empresas. Este bajo nivel del estadístico señala la necesidad de incorporar otras variables explicativas del comportamiento emprendedor para estimar correctamente dicha actitud en la población activa. A este respecto se sugiere la incorporación de variables tanto relativas al entorno -e.g., crecimiento económico del país, apoyo económico a la emprendeduría, facilidad de trámites administrativos, localización geográfica, cultura, etc.- como relacionadas con el individuo - e.g., creatividad, propensión a asumir riesgos, necesidad de logros, experiencia emprendedora, familia con empresa propia, etc. 
La influencia de los rasgos psicológicos en las actitudes hacia el empleo

\section{REFERENCIAS BIBLIOGRÁFICAS}

Acs, Z.J., Arenius, P., Hay, M. y Minniti, M. (2005). Global entrepreneurship monitor. 2004 Executive Report. USA: Babson \& London Business School.

Ardichvili, A. y Gasparishvili, A. (2003). Russian and Georgian entrepreneurs and non-entrepreneurs: a study of value differences. Organization Studies, 24(1), 29-46.

Arenius, P. y de Clercq, D. (2005). A network-based approach on opportunity. Small Business Economics, 24(3), 249-265.

Basu, A. (1998). An exploration of entrepreneurial activity among Asian Small Businesses in Britain. Small Business Economics, 10, 313-326.

Bauder, H. (2006). Origin, employment status and attitudes towards work: immigrants in Vancouver, Canada. Work, Employement and Society, 20(4), 709-729.

Becker, B.E. y Hills, S.M. (1981). Youth attitudes and adult labor market activity. Industrial Relations, 20, 60-70.

Becker, B.E. y Krzystofiak, F.J. (1982). The influence of labor market discrimination on locus of control. Journal of Vocational Behavior, 21, 60-70.

Begley, T.M. y Tan, W.L. (2001). The socio-cultural environment for entrepreneurship: a comparison between East Asian and Anglo-Saxon countries. Journal of International Business Studies, 32(3), 537-553.

Boone, C., Olffen, W.V. y Roijakkers, N. (2004). Selection on the road to a career: evidence of personality sorting in educational choice. Journal of Career Development, 31, 61-78.

Byrne, D. y Strobl, E. (2004). Defining unemployment in developing countries: evidence from Trinidad Tobago. Journal of Development Economics, 73, 465-476.

Capella, E. y Weinstein, R.S. (2001). Turning around reading achievement: predictors of high school students' academic resilence. Journal of Educational Psychology, 93(4), 758-771.

Coleman, M. y Deleire, T. (2003). An economic model of locus of control and the human capital investment decision. Journal of Human Resources, 38(3), 701721.

Cowling, M. y Hayward, R. (2000). Out of unemployemet. Birmingham: Research Centre for Industrial Strategy, Birmingham University.

Cowling, M. y Taylor, M. (2001). Entreprenerurial Women and Men: Two different species? Small Business Economics, 16, 167-175.

De Cuyper, N., de Jong, J., de Witte, H., Isaksson, K., Rigotti, T. y Schalk, R. (2008). Literature review of theory and re search on the psychological impact of temporary employment: Towards a conceptual model. International Journal of Management Reviews, 10(1), 25-51.

Felfe,J., Schmook, R., Schyns, B. y Six, B. (2008). Does the form of employment make a difference?-Commitment of traditional, temporary, and self-employed workers. Journal of Vocational Behaviour, 72, 81-94. 
Ferri, J. (2005). History lessons: the end of American exceptionalism. Journal of Economic Perspectives, 19, 199-215.

Frantz, R.S. (1980). Internal-external locus of control and labor market performance: empirical evidence using longitudinal survey data. A Quarterly Journal of Human Behavior, 17, 23-29.

Gabriel, S.A. y Rosenthal, S.S. (1996). Commutes, neighbourhood effects, and earnings: An analysis of racial discrimination and compensating differentials. Journal of Urban Economics, 40, 61-83.

Galvao Baptista, J.A. y Vidigal da Silva, J. (2004). The issue of micro-Enterprise financing in Cape Verde. Working Paper 05/04 CEFAG elaborado en Universidade de Évora y financiado por el Banco Central de Cabo Verde.

García Cabrera, A.M. y García Soto, M.G. (2008). Cultural differences and entrepreneurial behaviour: an intra-country cross-cultural analysis in Cape Verde. Entrepreneurship \& Regional Development, 20(5), 451-483.

Gibson, B. (2005). The transition to a globalized economy: poverty, human capital and the informal sector in a structuralist CGE model. Journal of Development Economies, 78, 60-94.

Groves, M.O. (2005). How important is your personality? Labor market returns to personality for women in the US and UK. Journal of Economic Psychology, 26, 827 841.

Guest, D. (2004). Flexible employment contracts, the psychological contract and employee outcomes: an analysis and review of the evidence. International Journal of Management Reviews, 5/6, 1-19.

Hanisch, K.A. (1999). Job loss and unemployment research from 1994 to 1998: A review and recommendations for research and intervention. Journal of Vocational Behavior, 55, 188-220.

Harper, M. (1991). The role of enterprise in poor countries. Entrepreneurship. Theory and Practice, 15(4), 7-11.

Kambourov, G. y Manovskii, I. (2002). Occupational Specificity of Human Capital. London: University of Western Ontario.

Kruss, G. (2004). Employment and employability: expectations of higher education resoponsiveness in South Africa. Journal of Education Policy, 19(6), 673689.

Latack, J.D. (1986). Coping with job stress: Measures and future directions for scale development. Journal of Applied Psychology, 71, 377-385.

Layard, R., Nickell, S. y Jackman, R. (2005). Unemployment: Macroeconomic Performance and the Labour Market. Oxford: Oxford University Press.

Lazarus, R.S. y Folkman, S. (1984). Stress, appraisal, and coping. New York: Springer.

Lee, J.S. y Hsin, P.L. (2004). Employee training and human capital in Taiwan. Journal of Wordl Business, 39, 362-376.

Lee, D.Y. y Tsang, E.W.K. (2001). The effects of entrepreneurial personality, background and network activities on ven- 
La influencia de los rasgos psicológicos en las actitudes hacia el empleo

ture growth. Journal of Management Studies, 38(4), 583-602.

Levenson, H. (1974). Activism and powerful others: distinction within the concept of external control. Journal of Personality Assessment, 38(4), 377-383.

Liem, R. (1992). Unemployed workers and their families: Social victims or social critics? En P. Voydanoff y L.C. Majka (Eds.), Families and Economic Distress (pp.131-151). Beverly Hills, CA. Sage.

Martínez García, M.F., García Ramírez, M. y Maya Jariego, I. (2002). Social support and locus of control as predictors of psychological well-being in Moroccan and Peruvian inmigrant women in Spain. International Journal of Intercultural Relations, 26, 287-310.

McClelland, D.C. (1961). The achieving society. Princenton: Van Nostrand Reinhold.

Mcquaid, R.W., Greig, M. y Adams, J. (2001). Unemployed job seeker attitudes towards potential travel-to-work times. Growth and Change, 32, 355-368.

Minniti, M., Bygrave, W.D. y Autio, E. (2006). GEM, Global entrepreneurship monitor. 2005 Executive Report. London, UK: London Business School and Babson Park.

Mitchell, R.K., Smith, B., Sewright, K.W. y Morse, E.A. (2000). Cross-cultural cognitions and the venture creation decision. Academy of Management Journal, 43(5), 974-993.

Moscarini, G. y Thomsson, K. (2007). Occupational and job mobility in the US.
Scandinavian Journal of Economics, 109(4), 807-836.

Mueller, S.L. y Thomas, A.S. (2000). Culture and entrepreneurial potential: a nine country study of locus of control and innovativeness. Journal of Business Venturing, 16, 51-75.

Mwamwenda, T. (1995). Job satisfaction among secondary school teachers in Transkei. South African Journal of Education, 15(2), 84-87.

Noorderhaven, N.G. y Tidjani, B. (2001). Culture, governance and economic performance: an explorative study with a special focus on Africa. International Journal of Cross Cultural Management, 1(1), 31-52.

Ogura, K. (2005). International comparison of atypical employment: Differing concepts and realities in industrialized countries. Japan Labor Review, 2, 5-29.

Omizo, M.M., Cubberly, W.W.y Omizo, S.A. (1985). The effect of rational-emotive education groups on self-concept and locus of control among learning disabled children. International Journal of Disability, Development and Education, 32(1), 13-19.

Ong, P. y Blumenberg, E. (1998). Job access, commute and travel burden among welfare recipients. Urban Studies, 35, 77-93.

Poirson, H. (2001). The impact of intersectoral labour reallocation on economic growth. Journal of African Economies, 10, 37-63.

Premeaux, S.F. y Bedeian, A.G. (2003). Breaking the silence: the moderating 
effects of self-monitoring in predicting speaking up in the workplace. Journal of Management Studies, 40(6), 1537-1562.

Reynolds, P.D., Bygrave, W.D., Autio, E., Cox, L.W. y Hay, M. (2002). Global entrepreneurship monitor. 2002 Executive report. USA: Babson \& London Business School and Kauffman Foundation.

Rotter, J.B. (1966). Generalized expectancies for internalized versus externalized reinforcement. Psychological Monographs, 80, whole 609.

Sanders, J. y de Grip, A. (2004). Training, task flexibility and the employability of low -skilled workers. International Journal of Manpower, 25(1), 73-89.

Schafgans, M.M.A. (2000). Gender wage differences in Malaysia: parametric and semiparametric estimation. Journal of Development Economics, 63, 351-378.

Slattery, J.P., Selvarajan T.T. y Anderson J.E. (2008). The influences of new employee development practices upon role stressors and work-related attitudes of temporary employees. The International Journal of Human Resource Management, 19(12), 2268-2293.

Spector, P.E., Cooper, C.L., Sánchez, J.I., O’Driscoll, M. y otros (2001). Do national levels of individualism and internal locus of control relate to wellbeing: an ecological level internal study. Journal of Organizational Behavior, 22, 815-832.
Stevens, M., Steffen, J., Dirroco, P. y David, R. (2007). The effects of adventure education on the locus of control of middle school and high school at-risk students. Research Quarterly for Exercise and Sport, 78(1), A104-A104.

Subramanian, D. (2008). Occupational mobility and internal labour markets: public sector workers' struggles in Bangalore. Internationaal Instituut voor Sociale Geschiedenis, 53, 425-457.

Thomas, A.S. y Mueller, S.L. (2000). A case for comparative entrepreneurship: assessing the relevance of culture. Journal of International Business Studies, 31(2), 287-301.

Todaro, M. P. (1997). Economic development beyond regulation: the informal economy in Latin America. MA: AddsionWesley. $6^{\text {th }}$ Edition.

Torka, N. y Schyns, B. (2007). On the transferability of 'traditional'satisfaction theory to non-traditional employment relationships temp agency work satisfaction. Employee Relations, 29, 440-457.

Verheul, I., Van Stel, A. y Thurik, R. (2006). Explaining female and male entrepreneurship at the country level. Entrepreneurship \& Regional Development, 18, 151-183.

Yeh, J.J.Y., Ko, J.J.R., Chang, Y.S. y Chen, C.H.V. (2007). Job stress and work attitudes between temporary and permanently employed nurses. Stress and Health, 23, 111-120. 